\title{
The Design and Implementation of Qt-based Cross-platform Video Conferencing Remote Control
}

\author{
Hua Yuan, Huixin Du \\ School of Computer Science and Engineering, South China University of Technology, Guangzhou, China \\ Email: hyuan@scut.edu.cn, qingyumini@foxmail.com
}

Received 2012

\begin{abstract}
In order to overcome the defects of the dedicated video conference remote control, this paper designs and implements a Qt-based cross-platform video conferencing remote control. The remote control not only implements a full range of video conferencing control functions with an easy-to-use visual interface; but also implement cross-platform feature to allow users to use remote control software on their own equipment. It is economic, convenient and stylish for users. This paper mainly describes the design and implementation of remote control system framework and cross-platform features. Finally, the result in the paper shows: This remote control is reliable and easy to use.
\end{abstract}

Keywords: Video Conferencing Remote Control; Cross-platform; Visualization

\section{Introduction}

As the direct display in front of users, video conferencing remote control is in close contact with the participants. It has a direct impact on the use of users. At present, most of video conferencing remote controls are dedicated infrared remote controls, such as Polycom. This kind of remote control sends the control instruction to terminals via infrared. Although the speed of its communication is fast, there are some shortcomings, such as less versatile, weak video conferencing supporting function, distance limitations and lack of visual interface.

In recent years, with the development of smart mobile devices, there are many attempts of developing remote control software on smart mobile devices [1, 2], such as FLPR Universal Remote made by App Cessory. An IOS product with FLPR Universal Remote connects to other appliances by infrared transmitter, and then downloads remote commands for 46000 different appliances. So the user can use such an IOS product to remote appliances. However, most of this remote control software are platform dependence and dedicated. They limit users' choices on the remote control hardware.

If we can generate remote control software, which can turn any smart mobile devices to a remote control by installing it, it will bring great convenience for users to start their video conferencing control. It will be economical and fashion to use such a remote control. Therefore, this paper presents a program about design and implementation the cross-platform video conferencing remote based on Qt. Qt is a cross-platform develop- ment framework. To ensure the realization of crossplatform remote control system, we need to develop remote control system based on Qt with some specialized design and coding work. The modular design is also needed for reducing the coupling of the system, so that the remote control system will have good scalability.

\section{Design of Remote Control System}

As an independent system, remote control is responsible for obtaining the relevant meeting information and controlling video conferencing terminal. So it needs to use multiple protocols to communicate with multiple objects. It may lead to the information handling confusion. Due to the diversity of the remote control function, multiinterface is used for user-friendly operation. And the implementation of cross-platform and scalability also asks for a modular structure. Therefore, the architecture design of remote control system should be based on modular as the standard.

The remote control system framework design is shown as Figure 1. The remote control system framework is divided into three layers: UI layer, logic layer and communication layer.

The UI layer is mainly used to provide users with a friendly visual interface. The interface module contains a number of user interfaces, such as login interface, conference list interface, meeting-place interface and terminal control interface.

Logic layer is similar to a dispatcher. It is responsible for processing the signal from the upper or lower layer 
and then sending the data of the signal to the corresponding module. It makes the UI layer and communication layer not need to consider whether their signal is correctly passed to the corresponding module and how to deal with. They only need to consider their own functions. The logic layer itself does not deal with data but only distribute the data. When the logic layer receives control signal from UI layer, logic layer calls corresponding slot to transfer the data to relevant communication module, so that the data transfer from UI is completed. The transfer from communication layer is similar to the transfer from UI layer. Communication layer just needs to send out a signal when it receives a data packet from the server or terminal. Logic layer will catch this signal by relevant slot and send out an update UI signal automatically to trigger the interface update.

As a key part of communication work, communication layer is responsible for establishing a connection, encapsulating and decapsulating data, sending and receiving packets. This layer contains two modules, server communication module and terminal communication module.

The server communication module is handling for the server-related communication work, such as meeting information acquisition, identity authentication, conferences login and logout. Because of the communication with server using two different protocols, this module also includes two sub-modules, Sip sub-module and Http sub-module. Sip protocol [3] is the basis of video conferencing systems. All the conference control data is transmitted through Sip protocol. Http protocol [4] is used to get those kinds of conference information whose data is huge. So that even in the face of bad hardware conditions, remote control can still get integrated and reliable data in high speed.

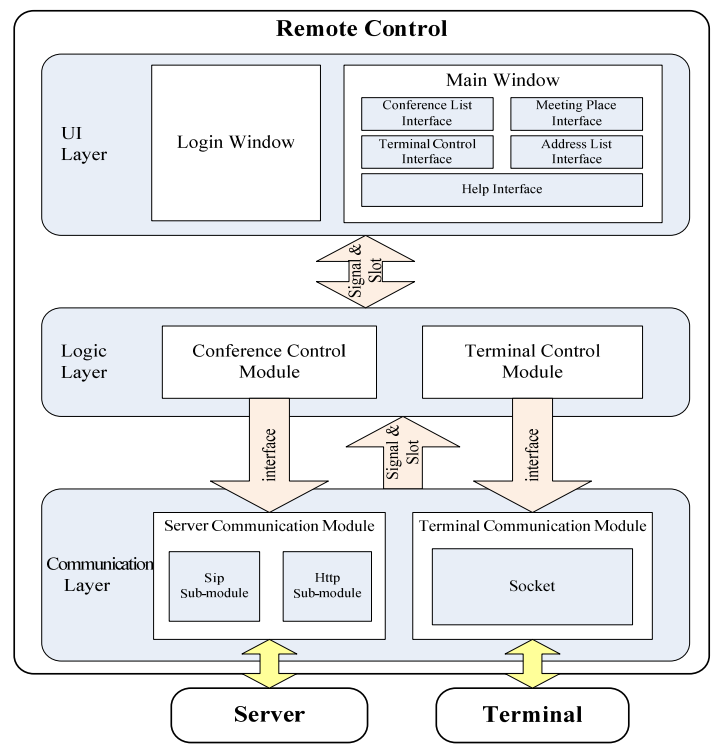

Figure 1. Framework design of remote control system.
Terminal communication module is used to handle all the communications for controlling terminal, including control of terminal audio and video stream, volume control, terminal mouse control and state synchronization. System uses Tcp socket connection for the communication between remote control and terminal. Json is used to encapsulate data. The Magic Packet module is used to send the magic packet to the LAN, in order to enable the wake-up function.

Signal and slot mechanism is used for the interface between layers. It is a Qt unique mechanism. It is designed specifically for communication between objects. Its big advantage is that both sides of communication don't need to know who the object communicates with. So using signal and slot mechanism as the interface increases cohesion and reduces coupling. It's a great help the system to implement architecture modular.

\section{Implement of Remote Control System}

The remote control in this paper uses a cross-platform development framework Qt and C++ programming language. Qt provides developers with the graphic related class libraries and the connection management class libraries such as QWidget and QNetwork Access Manager [5], using Qt can be easily implemented most of the functionality of the remote control. The Sip module of our remote control is based on the OSIP class library and is programming in $\mathrm{C}++$ language.

The workflow of the remote control is showed as Figure 2. When the user operates the interface of the remote control, the interface will send a signal for triggering the slot function in the logic layer. The slot function on logic layer will analyze this signal and send out a new signal which is bound with the corresponding communication module. As the signal and the slot function is mutually bound, the slot corresponding to the communication module will be able to successfully trigger, then the slot encapsulates the data and sends the data packet to the corresponding communication object.

In contrast, when the communication object sends a message to the remote control, the corresponding communication module receives and parses the packet, then sends out the signal which contains the relevant data. The logic layer receives the signal and triggers the slot function, and then the signal will be send to the UI layer by the slot function. When the interface components of the UI layer detects the signal which is bound to its own slot function, it will be updated automatically according to the signal data.

\section{Implement of Cross-platform Feature}

To implementation cross-platform feature, the crossplatform ideas should be implemented throughout the 

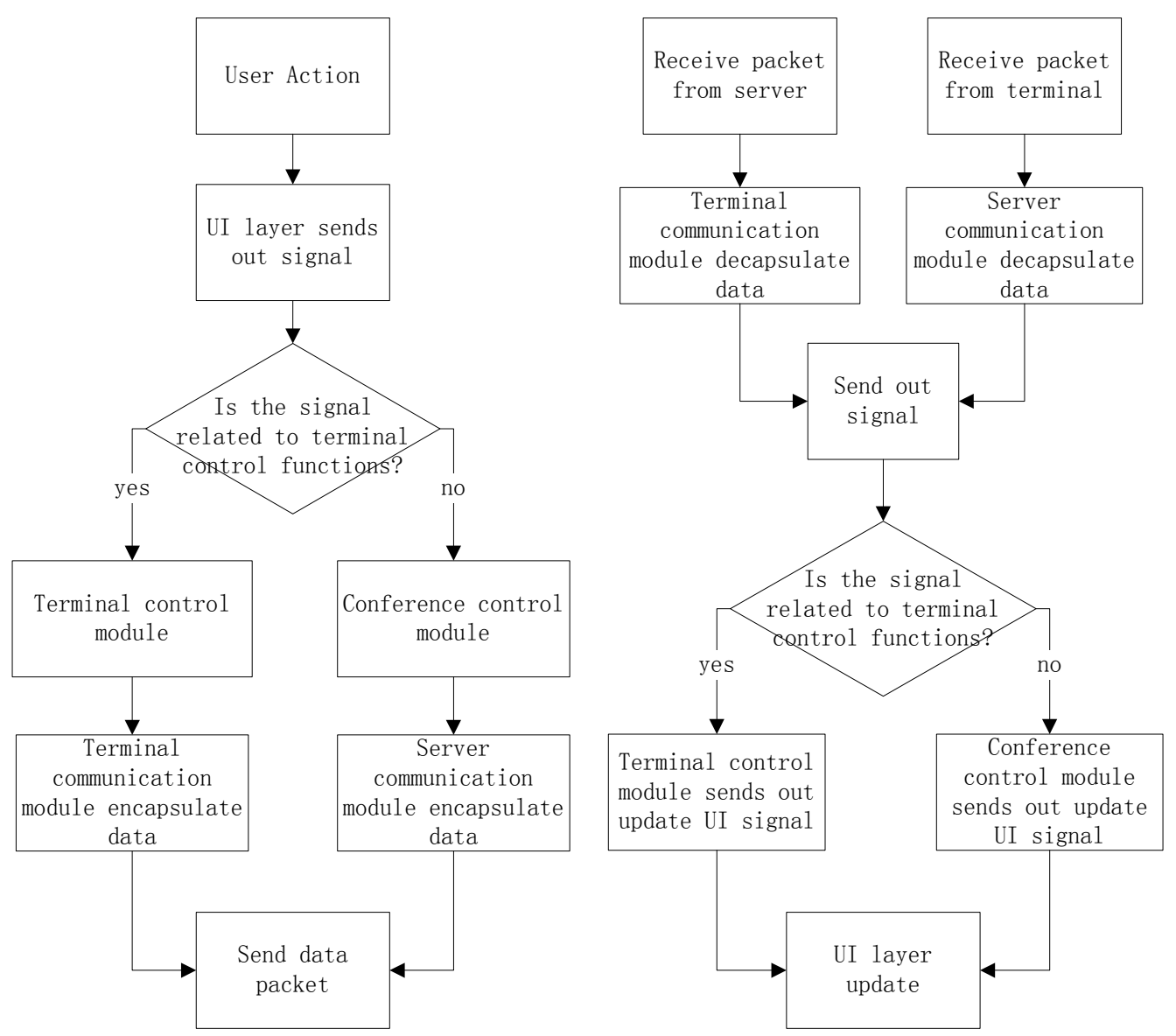

Figure 2. Workflow of the remote control system.

development cycle. In this paper, we realize the crossplatform characteristics by choosing a cross-platform language, designing, coding and compiling.

\subsection{Cross-platform Language}

This paper chooses Qt as a program development language. Qt is a high-level C + + based language. It has a good cross-platform feature, supporting for embedded Linux, Windows, Linux/X11, the Windows CE, Symbian, MeeGo and other embedded and PC operating systems [5]. Qt has a set of corresponding underlying class libraries for each supported operating system platform, but the interface of each is entirely consistent, so the program developed by Qt can be compiled and used under different operations. Qt can do as much as possible to help developer to implementation cross-platform features at the bottom.

\subsection{Code Modification for Cross-platform Feature}

Modify the framework and code according to the operation of the different platforms. As the previously mentioned, the Qt cross-platform framework has been im- plement the basic cross-platform functionality for the developers, but It can only guarantee to properly run the program on different platforms, cannot guarantee that no functional differences.

The Sip protocol is specifically designed for the multimedia conferencing; therefore, it contains all the basic conference control function. However, it maintains a same "seq num" on the server and the remote control for synchronization. When receiving a large amount of conferencing data on a remote control whose performance is poor, it may cause the server and the remote out of sync, resulting in the packet loss and return the error massage "500 Request out of Order". To avoid this, the HTTP module is added in the communication layer, it's used to receive the large amount of conferencing data such as conference list and member list.

\subsection{Adaptive Interface Design for Different Devices}

The Qt provides developers with a variety of platformstyle [6]: Windows, Motif, Mac etc. When developers do not have a certain style settings for the interface, the Qt will use the corresponding platform interface style. 
However, different interface styles may bring the risk of cross-platform interface differences. For the above reasons, most of the components use custom styles or redrew their appearance, in order to avoid different platforms to show differently. Qt provides the user with style description syntax which is similar with the CSS Style Sheet, so that user can set the components to custom style by using the "set Style Sheet" function in the code and set the style settings by using the style editing window which is provided by the Qt Designer[7].

The differences between the portable devices and PC equipment also cause operational differences, so we add the drag Action to avoid the double-click action. For example, when choosing the receive user's video stream, the design on PC is double-click the user to receive the video, however, if the system is ported to a portable device the user cannot operate. Therefore, we change the video receive operation to drag the user to the corresponding in the preview box. In this, regardless of the platform, user can very easy and intuitive to operate.

\subsection{Compiler}

Qt provides a tool - Qmake, which is specifically for transferring the platform-independent project code to the platform-dependent Makefile. Each project contains a .pro file for telling Qmake to create the details of the application make file, such as the list of header files and source files of the program, the specific configuration of the application, the additional libraries or additional include paths, etc. After the developer completed the coding, using the Qmake command will generate the corresponding Make file, make file.release, makefile.debug and the debug, release directory according to the. ro file. Makefile is the platform-dependent file, is used to define a series of rules to tell the platform compiler which files need to compile, even some complex operations. Makefile has two versions: debug and release. The debug version will be some additional processing, printing more $\log$ for debugging. The release version is generally used for the release of the program. It will turn off unnecessary log. But both are able to generate an executable program. Thus using Make file and the corresponding complier can generate the executable program of the corresponding platform in the debug and release directory. This process is shown in Figure 3.

Qt provides the integrated development environment Qt Creator which supports Window, Linux and Mac operating system. Qt Creator is not only to provide users with the code editor, file browser and class diagram tool, but also integrates Qmake build tool and the corresponding platform compiler. So, using Qt Creator will be able to complete all of the compiled work. For embedded platforms, the code cannot be compiled on the embedded platform, therefore, cross-compiler is used to complete the generation of the platform executable. Cross-compiler is to generate the executable code for the target platform on another platform. The remote control for embedded Linux platform is use the Scratchbox cross compiler.

\section{Testing Result}

Our remote control is a cross-platform for the PC platform and embedded platforms. The remote control can satisfy all the requirements for the video conferencing, and provides users with a rich and intuitive user interface, allowing users to operate smoothly without mouse, keyboard and other peripherals.

The meeting-place interface is the main control interface of the video conferencing, it contains three parts. From top to bottom, the first one is the preview window which is used to show the screen layout state of the current controlled terminal; the second part is the terminal member list which is used to show the state of member and provides a free drag and drop functionality between the preview window and the list of members; the last one is the control button bar provides the necessary operation of video conferencing.

The terminal control interface is used for connecting, waking up and closing the target terminal.

It can run on the PC platform and the embedded platforms, including Win XP, Vista, Win 7, Linux and embedded Linux operating system. The experiments show that the remote control can be installed and run in a variety of smart mobile devices such as smart phones, electronic tablet, laptop computers, etc.

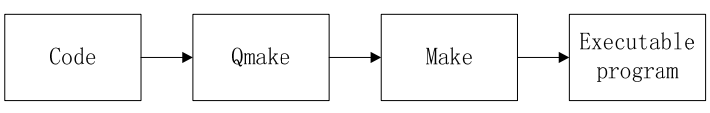

Figure 3. Compile process.

\section{Conclusions}

The remote control system developed in this paper has implemented the video conferencing functions and cross-platform feature. It can run on Win XP, Vista, Win 7, Linux and embedded Linux with no difference. Now it has been put into use in video conferencing systems.

\section{REFERENCES}

[1] Win8 Metro Testbed. http://www.splashtop.com/win8

[2] FLPR. http://www.newpotatotech.com/FLPR/flpr.html

[3] J. Rosenberg, "A Framework for Conferencing with the Session Initiation Protocol (SIP),” RFC 4353, 2006.

[4] A. S. Tanenbaum, Computer Networks, 4th ed., Tsinghua University, 2004, pp.651-656 
[5] Qt-Cross-platform Application and UI Framework. http://qt.nokia.com/

[6] H. D. Liu, J. W. Rui, Y. D. Yao and J. Wu, "Design and Implementation of Internationalized Graphical User Interface Based on Qt,” Journal of Chinese Information
Processing, Vol. 20, 2006, pp. 95-99.

[7] J. Blanchette and M. Summerfield, "C++ GUI Programming with Qt4,” 2nd., Publishing House of Electronics Industry, 2008. 